\title{
Duplex Nanostructured TiO2 Powder
}

\author{
Ahmed K. Al-Kamal ${ }^{1}$, Jafar F. Al-sharab ${ }^{1}$, Hadi Halim² ${ }^{2}$, Gang Xiong ${ }^{2}$, Bernard H. Kear ${ }^{1}$, Stephen D. \\ $\mathrm{Tse}^{2}$ \\ ${ }^{1}$ Department of Materials and Science Engineering, ${ }^{2}$ Department of Mechanical and Aerospace \\ Engineering, Rutgers University, Piscataway NJ, 08854
}

Titanium dioxide $\left(\mathrm{TiO}_{2}\right)$, is an attractive candidate material for a host of applications due to its exceptional properties, including high photocatalytic activity, hydrophilicity, stability, non-toxicity, and low cost [1]. Many techniques have been used to synthesize nanostructured $\mathrm{TiO}_{2}$-based powders, such as flame synthesis, sol-gel, laser ablation, and various wet chemical methods. More recently, the laser ablation method has received attention, because of the ability to precisely control particle size and distribution, as well as enabling easy collection of as synthesized nanoparticles [2]. In this paper, we describe the utilization of an Nd:YAG pulse laser, with 532-nm wavelength, and maximum pulse intensity of $500 \mathrm{~mJ}$, to induce controlled and reproducible decomposition of metalorganic precursors, with and without selective inorganic additives, in order to generate a new class of duplex nanostructured $\mathrm{TiO}_{2}$ powders. Here we describe preliminary results obtained by Laser Induced Precursor Pyrolysis (LIPP) of titanium tetraisopropoxide (TTIP).

Figure 1 shows aggregated $\mathrm{TiO}_{2}$ nanoparticles produced by LIPP of liquid TTIP precursor. To maximize the thermally induced decomposition rate, while preserving a rapid quench rate, the laser beam is focused just below the surface of the liquid precursor. As indicated the average nanoparticle size is $\sim 100 \mathrm{~nm}$, but within each particle the crystallite size is $<\sim 10 \mathrm{~nm}$. Evidently, extensive nanoparticle aggregation occurs during processing, indicative that even finer nanoparticles can be generated by modifying processing parameters to mitigate such aggregation. The XRD diffraction pattern, Figure 2 shows that the as synthesized particles have the metastable anatase structure. Similarly, the electron diffraction pattern shows that within each particle the anatase phase is preserved throughout, but close examination reveals that boundaries between the anatase nanocrystallites are often amorphous, as indicated by the continuous rings in the electron diffraction pattern. The broad peaks displayed by the XRD pattern give an estimated nanocrystallite size of $\sim 16 \mathrm{~nm}$, in agreement with the high resolution

TEM image, Figure 3-a. It is noteworthy that there is no evidence for the equilibrium rutile phase, which is often reported in the literature [3]. Further work is underway to determine the extent of doping the aggregated nanoparticles with other elements in order to optimize the photocatalytic activity.

References:

[1] Kinji Onoda, et al. Applied catalysis B: Environmental. 80(2008), pp. 277285

[2] Suiyuan Chen, et al. Applied surface Science. 283 (2013), pp.422-429.

[3] Thomas Rudin, et al. Microelectronic Engineering. 97 (2012), pp. 341-344.

[4] This research is partially funded by Higher Committee for Education Development in Iraq ( HCED). 

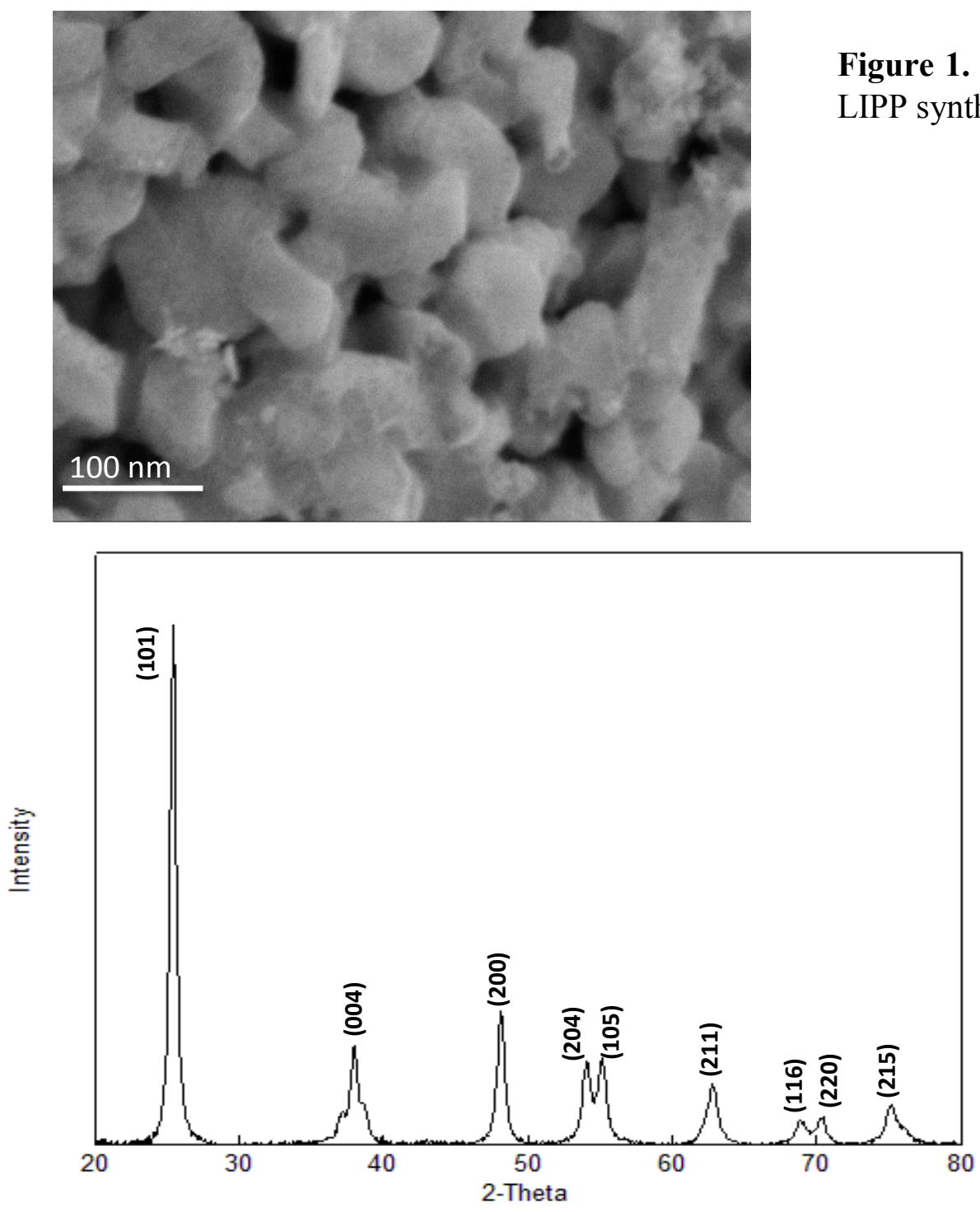

Figure 1. SEM micrograph of LIPP synthesized $\mathrm{TiO}_{2}$
Figure 2. XRD pattern of $\mathrm{TiO}_{2}$ powder. Peaks matches well with anatase phase (PDF\#97-018-3767)
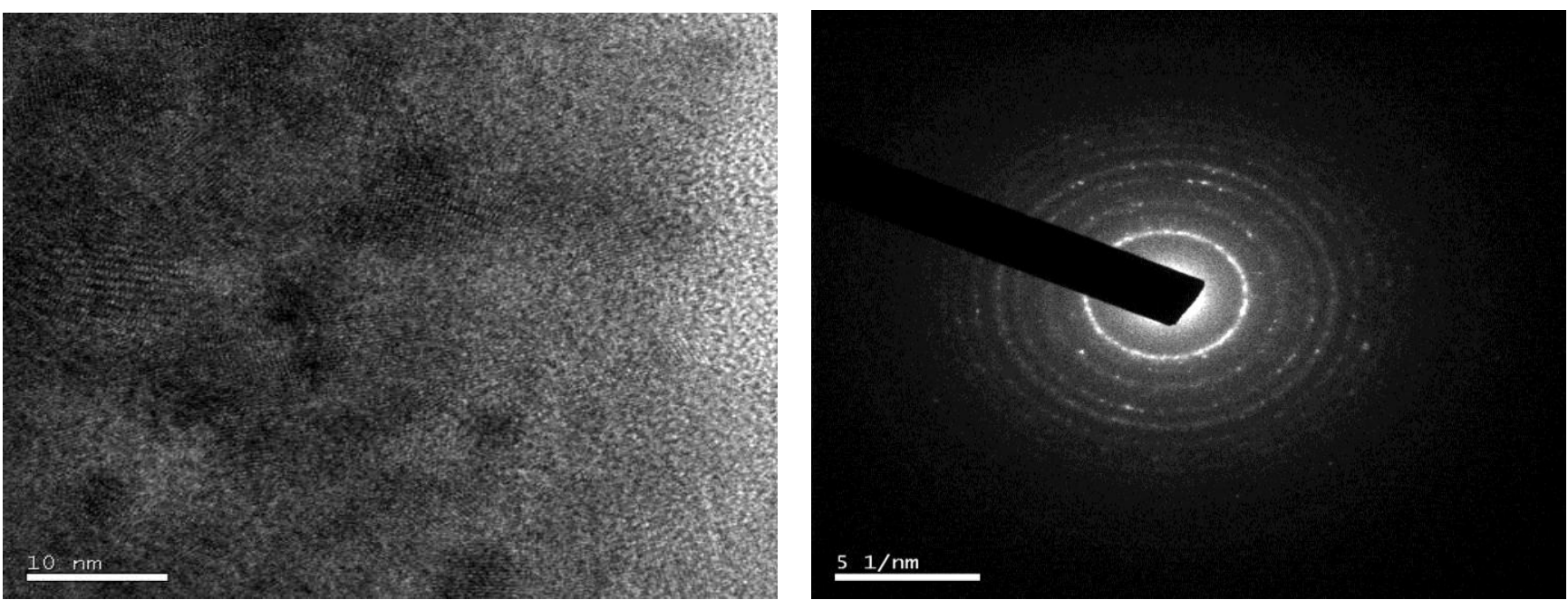

Figure 3. (a) HRTEM image, and (b) corresponding diffraction pattern of nanostructured $\mathrm{TiO}_{2}$ 